\title{
Application of active targeting nanoparticle delivery system for chemotherapeutic drugs and traditional/herbal medicines in cancer therapy: a systematic review
}

REVIEW

\author{
Nadda Muhamad' \\ Tullayakorn \\ Plengsuriyakarn ${ }^{1,2}$ \\ Kesara Na-Bangchang ${ }^{1,2}$ \\ 'Chulabhorn International College \\ of Medicine, Thammasat University, \\ Pathumthani, Thailand; ${ }^{2}$ Center of \\ Excellence in Pharmacology and \\ Molecular Biology of Malaria and \\ Cholangiocarcinoma, Chulabhorn \\ International College of Medicine, \\ Thammasat University, Pathumthani, \\ Thailand
}

\author{
This article was published in the following Dove Press journal: \\ International Journal of Nanomedicine
}

\begin{abstract}
Patients treated with conventional cancer chemotherapy suffer from side effects of the drugs due to non-selective action of chemotherapeutic drugs to normal cells. Active targeting nanoparticles that are conjugated to targeting ligands on the surface of nanoparticles play an important role in improving drug selectivity to the cancer cell. Several chemotherapeutic drugs and traditional/herbal medicines reported for anticancer activities have been investigated for their selective delivery to cancer cells by active targeting nanoparticles. This systematic review summarizes reports on this application. Literature search was conducted through PubMed database search up to March 2017 using the terms nanoparticle, chemotherapy, traditional medicine, herbal medicine, natural medicine, natural compound, cancer treatment, and active targeting. Out of 695 published articles, 61 articles were included in the analysis based on the predefined inclusion and exclusion criteria. The targeting ligands included proteins/peptides, hyaluronic acid, folic acid, antibodies/antibody fragments, aptamer, and carbohydrates/polysaccharides. In vitro and in vivo studies suggest that active targeting nanoparticles increase selectivity in cellular uptake and/or cytotoxicity over the conventional chemotherapeutic drugs and non-targeted nanoparticle platform, particularly enhancement of drug efficacy and safety. However, clinical studies are required to confirm these findings.
\end{abstract}

Keywords: active targeting, nanoparticles, ligands, chemotherapy, natural active compounds, cancer

\section{Introduction}

Cancer remains one of the major causes of deaths worldwide. In 2017, approximately 1.7 million new cases and 600 thousand deaths were estimated to occur in the USA. ${ }^{1}$ Most patients treated with conventional chemotherapy suffer from serious side effects due to non-selective action of chemotherapeutic drugs to normal cells. For a few decades, nanoparticles have been developed as a drug delivery system of various chemotherapeutic drugs to enhance drug efficacy and safety. ${ }^{2-4}$ Nanoparticles play an important role in increasing drug concentration in cancer cells by enhancing drug accumulation by passive and active targeting mechanisms as well as by decreasing drug efflux from cancer cells. The passive targeting nanoparticle is the mechanism by which the drugs leak from blood vessels supplying cancer cells and accumulate in the cells by enhanced permeability and retention (EPR) effect. ${ }^{5}$ The active targeting nanoparticles, on the other hand, target ligands conjugated on the surface of nanoparticles, resulting in increasing cellular uptake by receptor-mediated endocytosis and therefore increased
Correspondence: Kesara Na-Bangchang Graduate Studies, Chulabhorn International College of Medicine, Thammasat University (Rangsit Center), 99 Moo 18, Paholyothin Road, Klongnung, Klongluang, Pathumthani 12120 , Thailand Tel +66 25644400 ext I800

Fax +66 25644398

Email kesaratmu@yahoo.com 


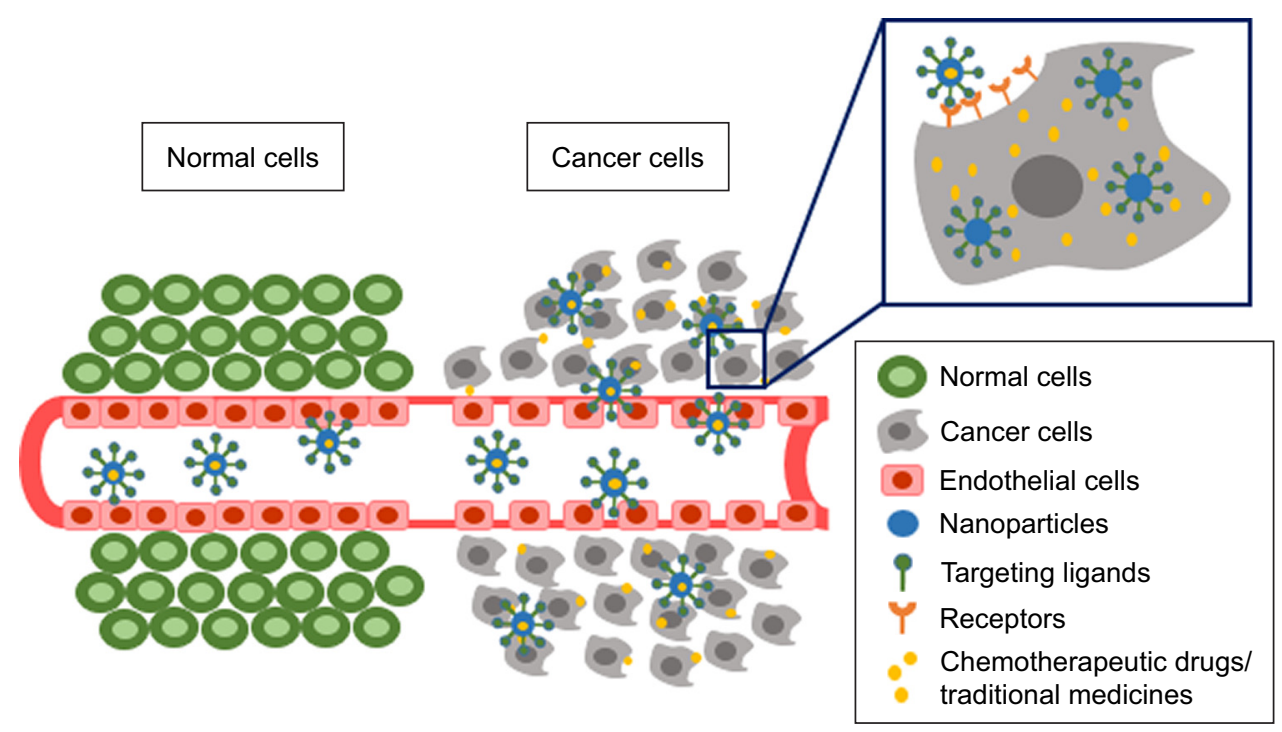

Figure I Passive targeting and active targeting mechanisms of nanoparticles.

drug accumulation in cancer cells. This mechanism relies on the interaction between tumor ligands conjugated on the surface of nanoparticles and cell-surface receptors or antigens on cancer cell surfaces (Figure 1). ${ }^{5}$ Nanoparticles acting via both mechanisms have been shown to increase drug concentration in cancer cells. Active targeting nanoparticles have been shown in various studies to be more efficient in increasing drug accumulation in cancer cells and therefore play important role not only in modern cancer chemotherapy, but also in cancer therapy with traditional/ herbal medicines. ${ }^{6-11}$ A number of nanoparticle formulations derived from these active compounds have been developed for active targeting purpose to improve anticancer efficacy and to reduce side effects. The objective of this current review is to summarize the research articles relating to the application of active targeting nanoparticles delivering system for chemotherapeutic drugs derived from chemical synthesis as well as natural sources.

\section{Materials and methods Study selection and inclusion and exclusion criteria}

This systematic review was conducted through the search from PubMed database up to March 2017. The following keywords were used: nanoparticle, chemotherapy, traditional medicine, herbal medicine, natural medicine, natural compound, cancer treatment, and active targeting. Inclusion criteria for selection of the searched articles were 1) articles in full text and written in English; 2) articles with in vitro or in vivo investigations of effects of nanoparticles delivering chemotherapeutic drugs or traditional/herbal medicines on efficacy and/or safety; and 3) articles with investigations of targeting and receptor/antigen. The articles with insufficient data for extraction or those with application for radiotherapy, gene therapy, photodynamic therapy, or for diagnostic purpose, or duplicate articles, or review articles were excluded from the analysis.

\section{Data extraction and collection}

The titles and abstracts of articles searched from PubMed database using the above mentioned keywords were initially screened to obtain relevant original research articles according to the eligibility criteria. Thereafter, the full texts of all relevant articles were carefully examined in details to confirm their compliance with the defined eligibility criteria. The studies of active targeting nanoparticles applied for both chemotherapeutic drugs and traditional/herbal medicines for cancer were classified according to the types of targeting ligands.

\section{Results Study description}

Twenty out of 695 research articles were initially excluded from the analysis during title screening for duplicate articles. The titles together with abstracts of the remaining articles were further checked for eligibility criteria and a total of 597 articles were excluded from the analysis. Finally, 61 out of 78 articles were included in the analysis, 17 articles being excluded due to unclear/inadequate information. The flow diagram of the search process is presented in Figure 2, and the 


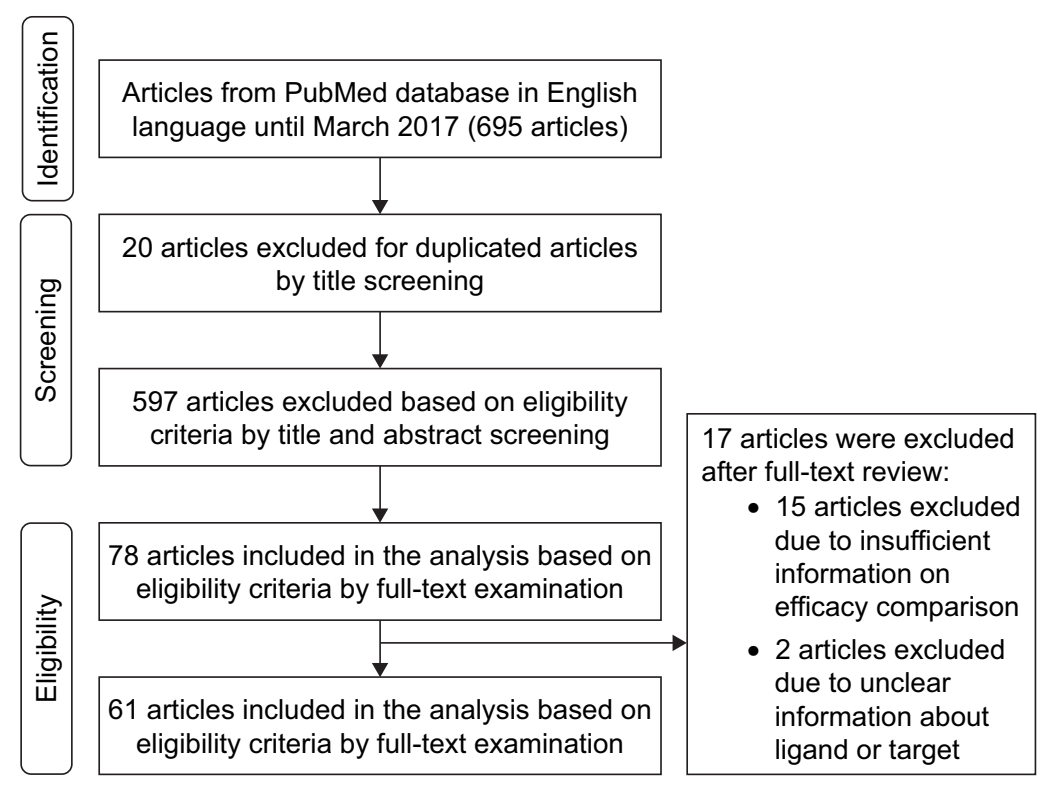

Figure 2 Flow diagram showing the different phases of the systematic review.

effects of active targeting nanoparticles delivering modern chemotherapeutic drugs and traditional/herbal medicines for cancer are summarized in Tables 1 and 2.

Of the 61 articles included in the analysis, 54 (88.5\%) investigated nanoparticles delivering modern chemotherapeutic drugs; the majority was doxorubicin (40.7\%), followed by paclitaxel (8.5\%). Types of targeting ligand platforms used included proteins or small peptides (15 articles), hyaluronic acids (HAs; 10 articles), folic acids ( 9 articles), antibodies (5 articles), aptamers (5 articles), carbohydrates or polysaccharide ( 5 articles), and other molecules (5 articles). Seven articles (11.5\%) investigated nanoparticles delivering traditional/herbal medicines; the majority was curcumin (42.9\%). The ligand platforms used were proteins or small peptides ( 2 articles), HA (1 article), folic acid (1 article), antibody (1 article), aptamer (1 article), and other molecule (1 article).

\section{Discussion}

\section{Ligands for nanoparticle platform}

\section{Proteins or small peptides}

Various types of proteins or small peptides were used to conjugate on the surface of nanoparticles to improve selectivity of chemotherapeutic drugs or traditional/herbal medicines to cancer cells. Transferrin, a serum glycoprotein, was one of the widely used targeting ligands. It plays a role in transferring iron from blood stream into the cells by binding to transferrin receptor on the cell surface. Upregulation of transferrin receptor has been reported in metastatic and drug-resistant cancer cells. ${ }^{67}$ The transferrin-conjugating nanoparticles delivering chemotherapeutic drugs have been shown to improve cellular uptake of the drugs by cancer cells and enhance in vitro and in vivo cytotoxicity. For instance, the transferrin-conjugated polyethylene glycol (PEG) nanoparticle delivering hydroxycamptothecin was shown to provide longer retention time of drug in blood circulation, higher drug accumulation in cancer cells, and higher in vivo growth inhibitory activity against S180 tumor compared with non-targeted nanoparticles. ${ }^{16}$ In the study of transferrinconjugated chitosan-PEG nanoparticles delivering paclitaxel, the targeted nanoparticles also exhibited higher cytotoxic activity to transferrin-overexpressing human non-small cell lung cancer cells (HOP-62). The respective half-maximal inhibitory concentrations $\left(\mathrm{ICs}_{50}\right.$ ) were $0.3 \mu \mathrm{M}$ and $2.0 \mu \mathrm{M} .{ }^{17}$ Apart from transferrin, arginine-glycine-aspartic acid (RGD) peptide has been used as targeting ligand to conjugate on the surface of nanoparticles to specifically target integrin $\alpha_{\mathrm{v}} \beta_{3}$ receptor. This receptor is expressed on the surface of tumor vessels and various types of cancer cells and plays important roles in tumor growth promotion, metastasis, and angiogenesis. ${ }^{18}$ A number of RGD-conjugated nanoparticles delivering chemotherapeutic drugs or traditional/herbal medicines have been developed and demonstrated to promote their delivery to the cancer cells. The cyclic arginine-glycine-aspartic acidtyrosine-lysine $\mathrm{c}(\mathrm{RGDyK})$-conjugated poly(trimethylene carbonate)-PEG micellar nanoparticle delivering paclitaxel was shown to enhance cytotoxic activity of the drug to integrin $\alpha_{v} \beta_{3}$-overexpressing human glioblastoma cells 


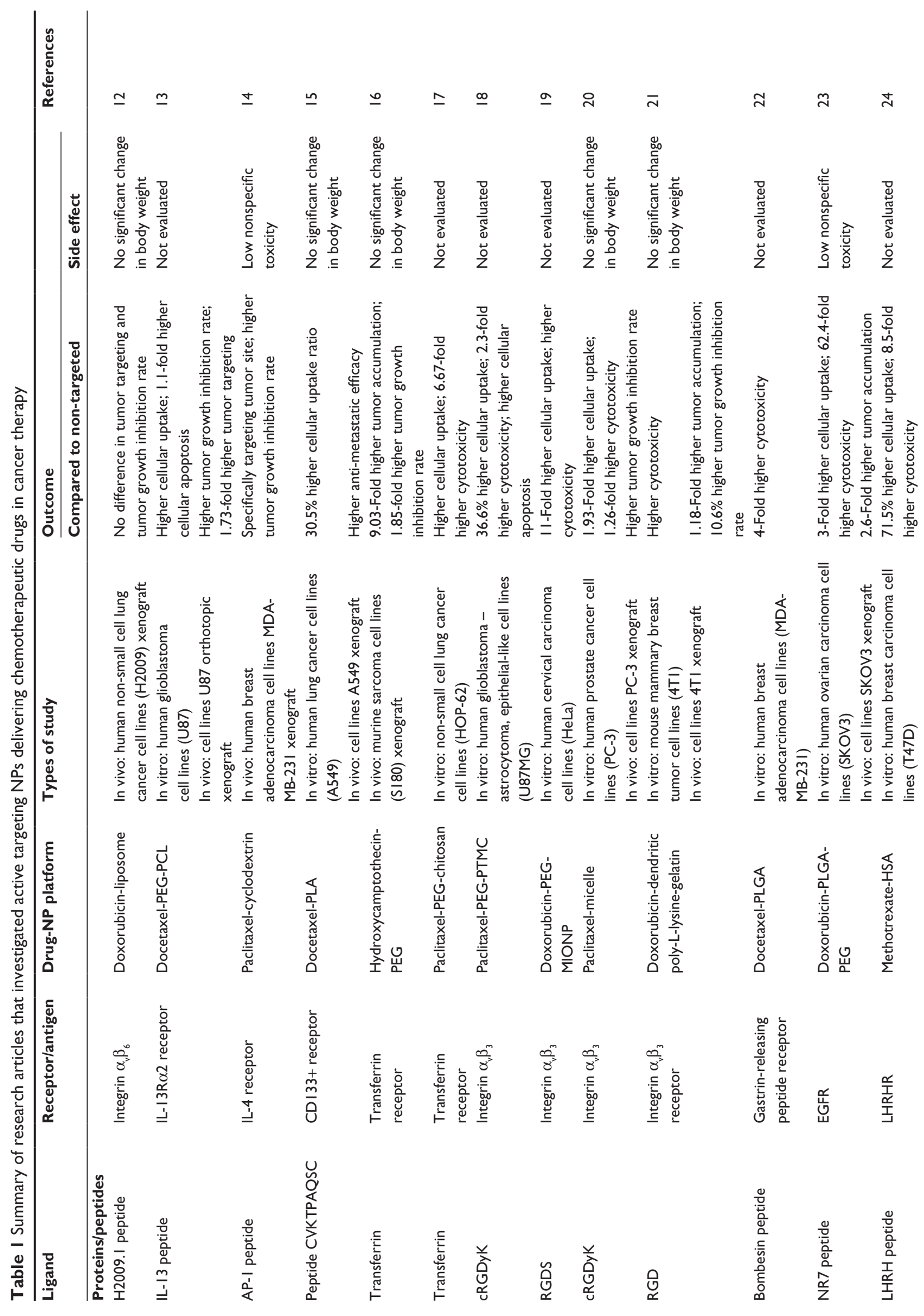




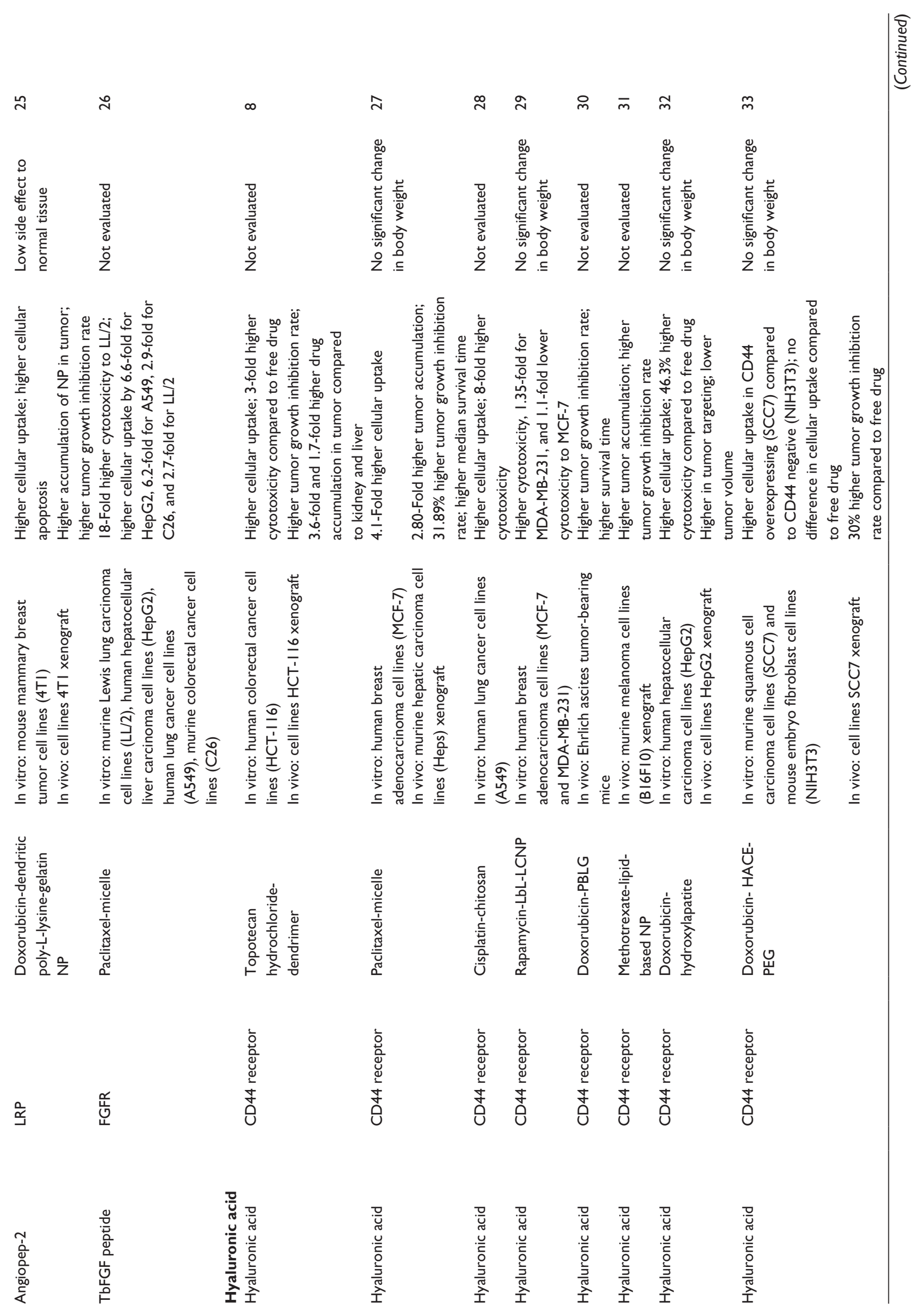




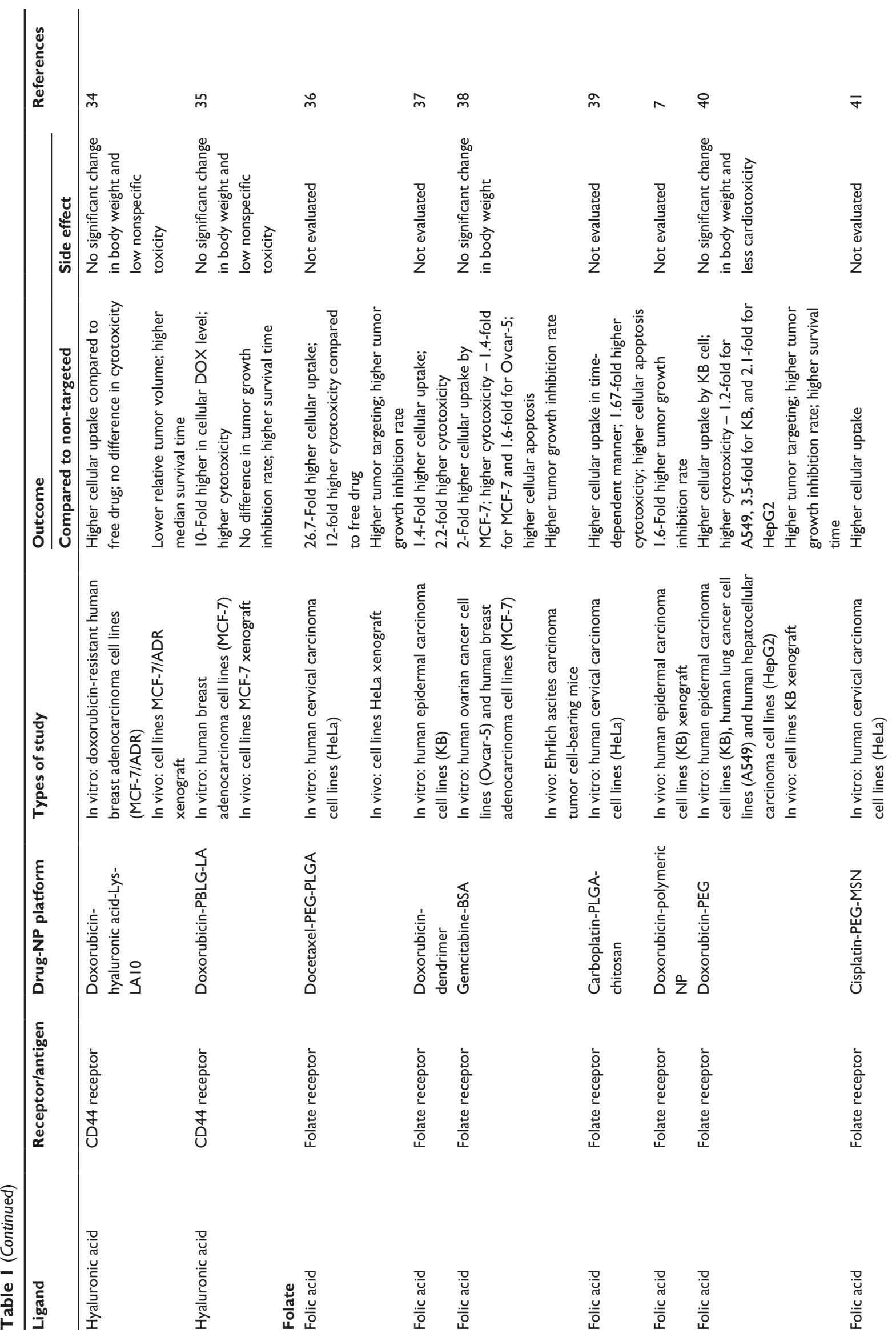



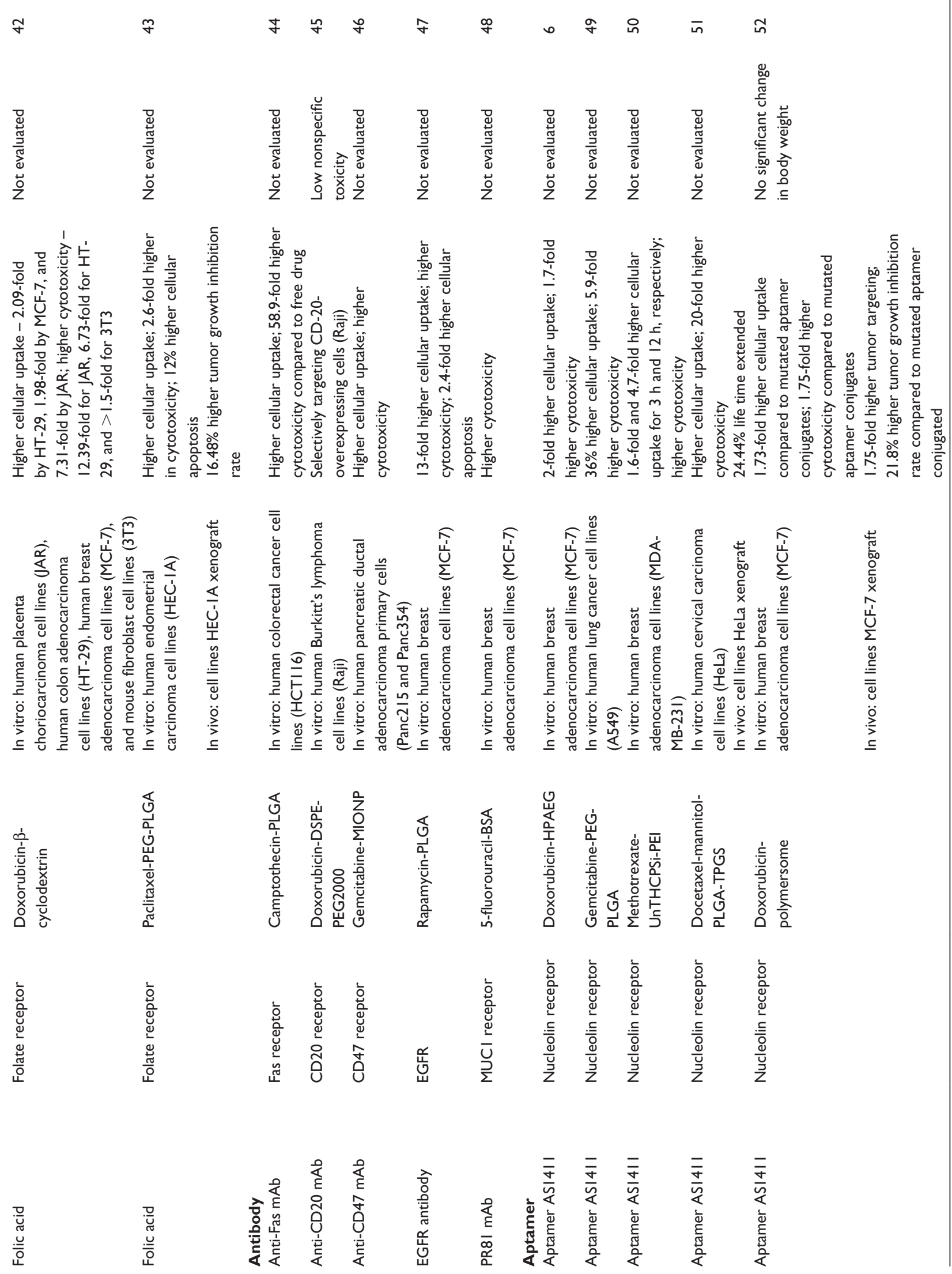


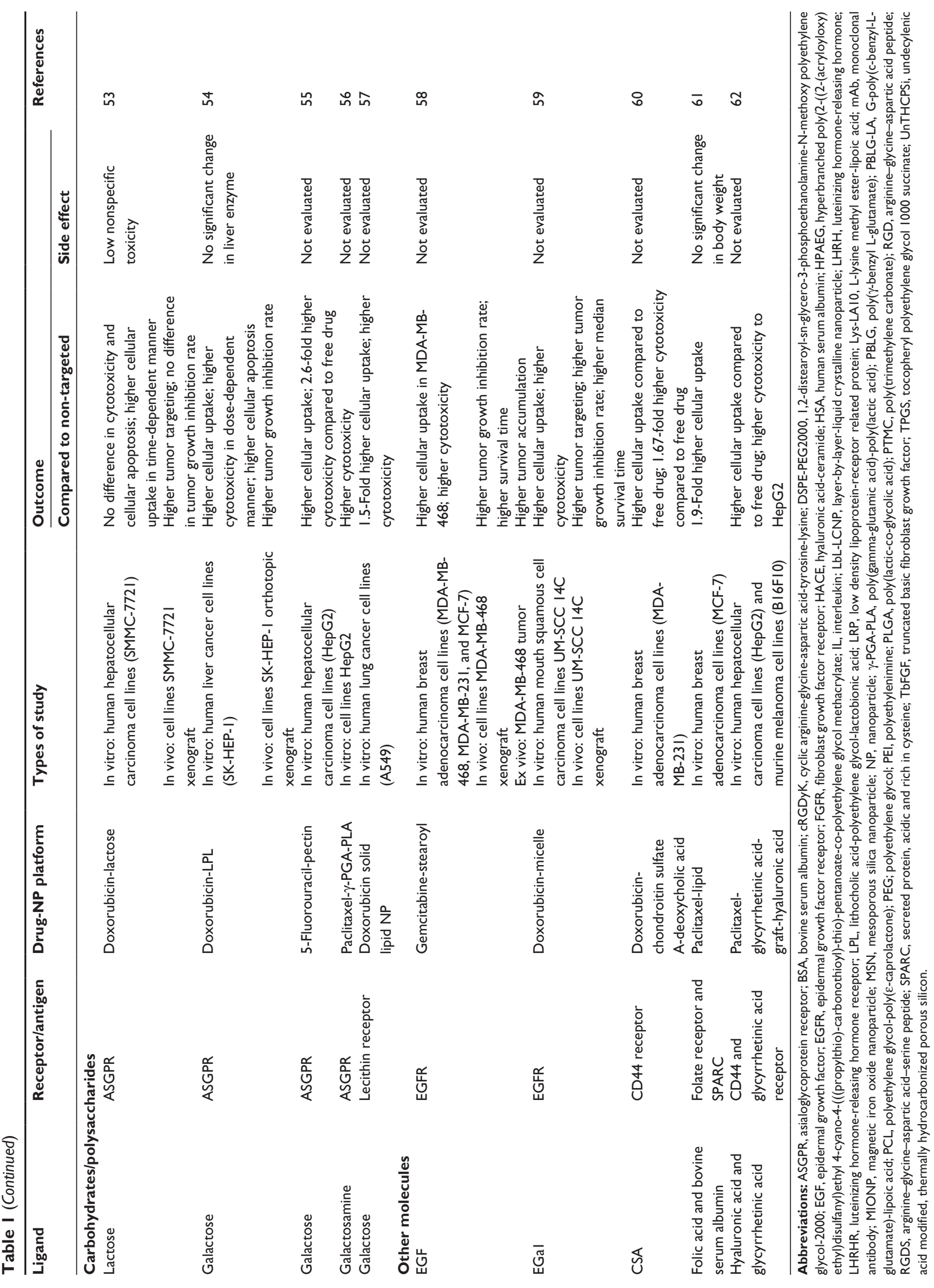




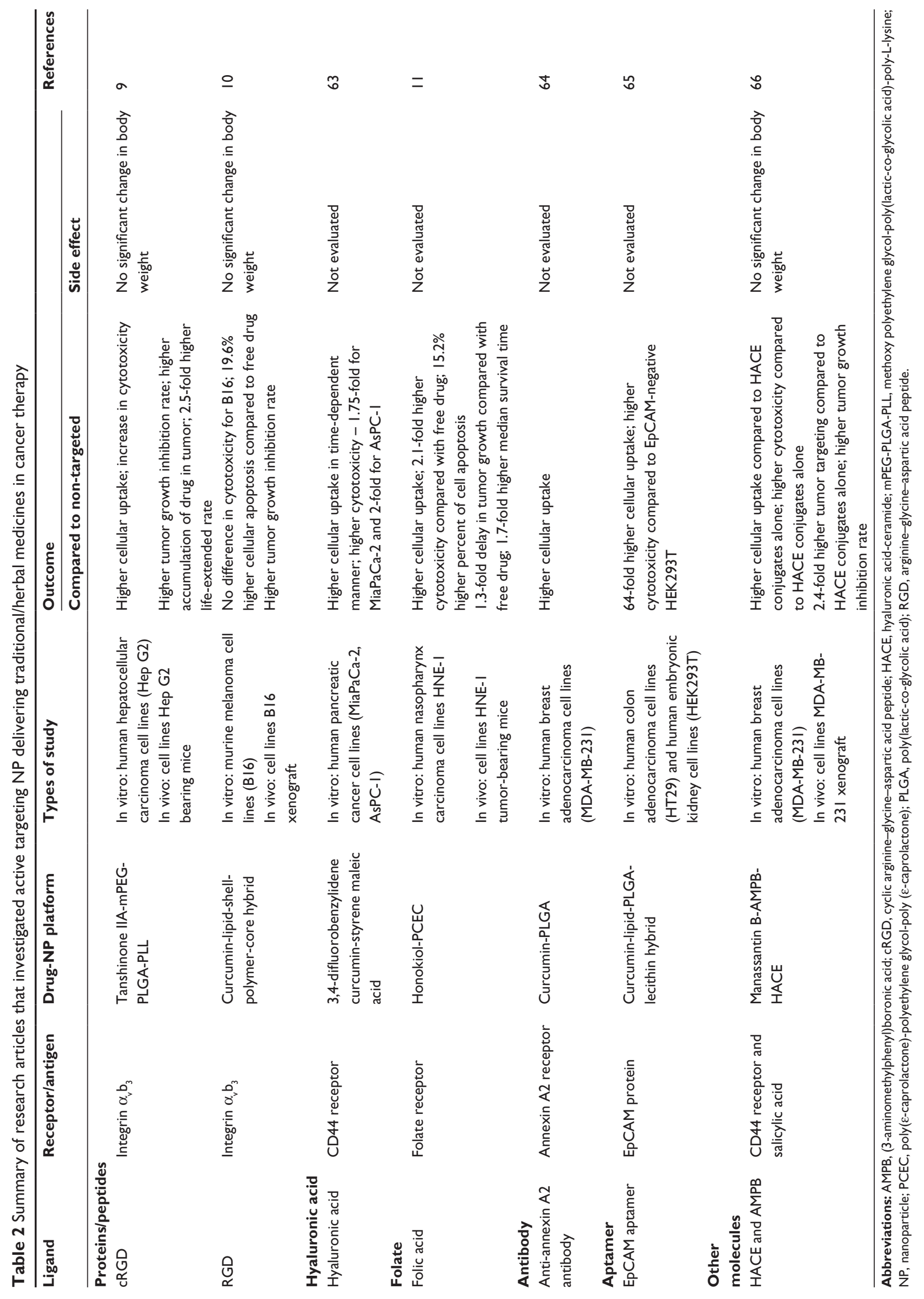


(U87MG) compared with non-targeted nanoparticles and free drugs (mean $\mathrm{IC}_{50}: 0.022 \mu \mathrm{g} / \mathrm{mL}, 0.051 \mu \mathrm{g} / \mathrm{mL}$, and $0.058 \mu \mathrm{g} / \mathrm{mL}$, respectively). The targeted nanoparticles also exhibited greater activity on cell apoptosis $(11.23 \%$ vs $8.31 \%$ and $8.03 \%$ vs $5.38 \%$ inhibition, for early and late apoptosis, respectively). The percentages (mean values) of free drug were $6.67 \%$ and $4.32 \%$, respectively. In addition, cellular drug uptake by U87MG cells was significantly increased. ${ }^{18}$ Superior cytotoxic potency against integrin $\alpha_{\mathrm{v}} \beta_{3}$-overexpressing human cervical carcinoma cells (HeLa) together with increased cellular uptake was also demonstrated with RGD-conjugated magnetic iron oxide nanoparticles (MIONPs)-PEG delivering doxorubicin compared with free drug and non-targeted MIONPs. ${ }^{19}$ In another study, improved cytotoxic activity by the cRGDyK-conjugated poly(2-ethyl2-oxazoline)-poly(D,L-lactide) nanoparticles delivering paclitaxel over the non-targeted nanoparticles and free drug was reported (mean $\mathrm{IC}_{50}: 51.16 \mathrm{ng} / \mathrm{mL}, 64.53 \mathrm{ng} / \mathrm{mL}$, and $62.95 \mathrm{ng} / \mathrm{mL}$, respectively). The enhanced activity was through direct targeting of the integrin $\alpha_{\mathrm{v}} \beta_{3}$-overexpressing prostate cancer cells (PC-3), as well as increasing of cellular uptake by PC-3 cells. Moreover, the targeted nanoparticle was also shown to enhance in vivo tumor growth inhibition rate in PC-3 tumor-bearing mice. ${ }^{20}$

Other types of peptides that have been applied for conjugation on the surface of nanoparticles to increase selectivity of chemotherapeutic drugs to cancer cells include bombesin peptide-conjugated poly(lactic-co-glycolic acid) (PLGA) and NR7 peptide-conjugated PLGA-PEG nanoparticles. Bombesin-conjugated nanoparticles delivering docetaxel specifically bind to gastrin-releasing peptide receptor, which is overexpressed on cell surfaces of prostate, breast, ovarian, pancreatic, and colorectal cancers. ${ }^{22,68}$ This targeted nanoparticle was shown to enhance cytotoxic activity of the drug to the gastrin-releasing peptide receptor overexpressing human breast cancer cells (MDA-MB-231) compared with non-targeted nanoparticles (mean $\mathrm{IC}_{50}: 35.53 \mathrm{ng} / \mathrm{mL}$ and $142.23 \mathrm{ng} / \mathrm{mL}$, respectively). ${ }^{22}$ The NR7 peptide-conjugated PLGA-PEG nanoparticle delivering doxorubicin was used for specific drug binding to epidermal growth factor receptor (EGFR) on the cancer cell surface. ${ }^{23}$ The EGFR is a known receptor that is overexpressed on various types of cancer cell surfaces including head and neck, renal, ovarian, breast, and non-small-cell lung cancer. ${ }^{47,69}$ Activation of this receptor results in inhibition of cell apoptosis, promotion of cell proliferation, triggering of angiogenesis, and enhancement of tumor survival and metastasis. Therefore, inhibition of the function of this receptor would be expected to benefit cancer treatment. The NR7 peptide-conjugated PLGA-PEG nanoparticles exhibited higher cytotoxic activity against human ovarian carcinoma cells (SKOV3) compared with non-targeted nanoparticles (mean $\mathrm{IC}_{50}: 0.05 \mu \mathrm{g} / \mathrm{mL}$ and $3.12 \mu \mathrm{g} / \mathrm{mL}$, respectively). ${ }^{23}$ Although most studies demonstrated satisfactory outcomes of peptide- or protein-conjugated nanoparticles on targeting cancer cells, one study reported that H2009.1 peptide-conjugated liposome delivering doxorubicin to cancer cells expressing integrin $\alpha_{v} \beta_{6}$ receptor could not improve the efficacy of the drug. This was due to the liposome platform preventing the targeting ligand from binding to the receptor on the cancer cell surface, and resulted in relatively low drug accumulation in cancer cells. ${ }^{12}$

\section{Hyaluronic acid}

HA is a negatively charged linear glycosaminoglycan that consists of D-glucuronic acid and N-acetyl-D-glucosamine. It can specifically bind to CD44 receptor that is overexpressed on the cell surface of various types of cancer including lung, breast, colon, prostate, gastric, and head and neck cancers. ${ }^{70}$ $\mathrm{HA}$ is a widely used targeting ligand to conjugate on the surface of nanoparticles to improve selectivity of chemotherapeutic drugs to cancer cells and enhance drug efficacy and safety. In one study, HA with the two molecular weights, ie, $9.5 \mathrm{kDa}$ and $35 \mathrm{kDa}$, was used to conjugate polymeric micelles delivering paclitaxel. The conjugate using $9.5 \mathrm{kDa}$ HA was found to effectively increase drug cellular uptake by CD44-overexpressing human breast adenocarcinoma cells (MCF-7) compared with $35 \mathrm{kDa}$ HA. In murine hepatic carcinoma (Heps), it also exhibited tumor growth inhibition at a higher rate and greater accumulation at the tumor site compared with other nanoparticle formulations. ${ }^{27}$ These results suggest that the molecular weight of HA directly influenced the efficacy of drug-loaded active targeting nanoparticles. The HA-conjugated chitosan nanoparticle delivering cisplatin was shown to increase drug cellular uptake by CD44-positive human lung cancer cells (A549) and effectively enhance cytotoxic activity of the drug, compared with non-targeted nanoparticles. ${ }^{28}$ The HA-conjugated liquid crystalline nanoparticle delivering rapamycin was reported to increase cytotoxic activity of the drug to CD44-overexpressing human breast adenocarcinoma cells (MDA-MB-231) compared with non-targeted nanoparticles (mean $\mathrm{IC}_{50}: 18 \mu \mathrm{g} / \mathrm{mL}$ and $24.3 \mu \mathrm{g} / \mathrm{mL}$, respectively). Moreover, the targeted nanoparticles also enhanced in vivo tumor growth inhibition rate in Ehrlich ascites tumor-bearing mice. ${ }^{29}$

For traditional/herbal medicines, HA has also been used for conjugation on the surface of nanoparticles delivering 
3,4-difluorobenzylidene curcumin resulting in increased cellular uptake and cytotoxic activity of the drug against human pancreatic cancer cells (MiaPaCa-2 and AsPC-1) compared with non-targeted nanoparticles (mean $\mathrm{IC}_{50}: 140 \mathrm{nM}, 160 \mathrm{nM}$, and $245 \mathrm{nM}$, respectively). Interestingly, when the CD44 receptor was blocked by free soluble HA, the cytotoxic activity to MiaPaCa-2 cells was comparable between the targeted and non-targeted nanoparticles (mean $\mathrm{IC}_{50}: 234 \mathrm{nM}$ and $245 \mathrm{nM}$, respectively). ${ }^{63}$ These results confirm that targeting ligand-conjugated nanoparticles enhances drug efficacy by improving cellular uptake through receptor-mediated endocytosis mechanism.

\section{Folate}

Folate or vitamin B9 is a stable and poorly immunogenic water-soluble vitamin. It is essential for DNA synthesis and replication, methylation, cell division and growth, and cell survival, particularly in rapidly dividing cells or cancer cells. ${ }^{71}$ Folic acid receptor is overexpressed on several cancer cell surfaces including ovarian, cervical, breast, lung, kidney, colorectal, and brain cancers. ${ }^{71}$ Using folic acid as cancer cell targeting of chemotherapeutic drug nanocarriers has been demonstrated in various studies to improve drug efficacy and safety profiles. The folic acid-conjugated PEG-PLGA nanoparticle delivering docetaxel was shown to increase drug cellular uptake by human cervical carcinoma cells (HeLa) with enhanced cytotoxic activity compared with free drug (mean $\mathrm{IC}_{50}: 0.69 \mu \mathrm{g} / \mathrm{mL}$ and $8.29 \mu \mathrm{g} / \mathrm{mL}$, respectively). It also significantly inhibited tumor growth in HeLa tumor-bearing mice. ${ }^{36}$ The folic acid-conjugated albumin nanoparticle delivering gemcitabine was shown to enhance cytotoxic activity of the drug against folic acid receptor-overexpressing human breast adenocarcinoma cells (MCF-7) compared with non-targeted nanoparticles (mean $\mathrm{IC}_{50}: 0.175 \mu \mathrm{M}$ and $0.240 \mu \mathrm{M}$, respectively). Similarly, in folic acid receptor-overexpressing human ovarian cancer cells (Ovcar-5), the targeted nanoparticles exhibited superior cytotoxic activity over the non-targeted nanoparticles (mean $\mathrm{IC}_{50}: 0.173 \mu \mathrm{M}$ and $0.279 \mu \mathrm{M}$, respectively). On the other hand, activity of the targeted nanoparticles was found similar to that of non-targeted nanoparticles against folate receptor expressing human pancreatic cancer cells (MIAPaCa-2) (mean $\mathrm{IC}_{50}: 0.166 \mu \mathrm{M}$ and $0.169 \mu \mathrm{M}$, respectively). ${ }^{38}$ In one study, the folic acid-conjugated PEG-PLGA nanoparticle delivering paclitaxel was shown to increase drug cellular uptake by folic acid-overexpressing human endometrial carcinoma cells (HEC-1A) with superior cytotoxic activity over the non-targeted nanoparticle (mean $\mathrm{IC}_{50}: 3.43 \mu \mathrm{g} / \mathrm{mL}$ and $8.81 \mu \mathrm{g} / \mathrm{mL}$, respectively). Moreover, it also produced significantly higher cell apoptotic activity compared with non-targeted and free drug $(35.94 \%, 23.97 \%$ and $19 \%$, respectively). In vivo, it produced significant tumor growth inhibition in HEC-1 A tumor-bearing mice ${ }^{43}$ For traditional/ herbal medicines, folic acid-conjugated poly(epsiloncaprolactone)-PEG-poly (epsilon-caprolactone) nanoparticle delivering honokiol, a traditional Chinese medicine, was shown to increase compound cellular uptake by folic acidoverexpressing human nasopharynx carcinoma cells (HNE-1) with enhanced cytotoxic activity compared with free drug (mean $\mathrm{IC}_{50}: 18.41 \mu \mathrm{g} / \mathrm{mL}$ and $38.59 \mu \mathrm{g} / \mathrm{mL}$, respectively). Furthermore, the targeted nanoparticles also resulted in significant cell apoptotic activity compared with non-targeted nanoparticles ( $86.07 \%$ and $70.89 \%$, respectively) and prolongation of median survival time compared with non-targeted nanoparticles and free drug (median survival time: 57.5 days, 42.5 days, and 34 days, respectively). ${ }^{11}$

\section{Antibodies or antibody fragments}

Antibodies or antibody fragments are one of the first targeting ligands used for conjugation on the surface of nanoparticles to target cancer cells due to their potential to specifically bind to antigens or receptors on cancer cell surfaces with high affinity. Various types of antibodies or antibody fragments have been used as targeting agents including anti-CD20 monoclonal antibody, anti-CD47 monoclonal antibody, EGFR antibody, and anti-Fas monoclonal antibody. These targeted nanoparticles have been shown to improve cellular uptake by cancer cells and enhance cytotoxic activity of the drugs to cancer cells. For instance, anti-CD20 monoclonal antibody-conjugated 1,2-distearoyl-sn-glycero-3-phosphoethanolamine-N-methoxypolyethylene-glycol-2000 delivers doxorubicin active carbon nanoparticles to the target CD20 receptor. It exhibited higher cytotoxic activity against CD20positive human Burkitt's lymphoma cells (Raji) compared with non-targeted nanoparticles. ${ }^{45}$ The anti-CD47 monoclonal antibody-conjugated iron oxide magnetic nanoparticle delivering gemcitabine to the target CD47 receptor was shown to increase drug cellular uptake by human pancreatic ductal adenocarcinoma cells (Panc215 and Panc354). Their cytotoxic activity was also significantly enhanced. ${ }^{46}$ The EGFR antibody-conjugated PLGA nanoparticle delivering rapamycin was shown to exhibit higher cellular uptake by EGFR-overexpressing human breast adenocarcinoma cells (MCF-7) with enhanced cell apoptotic activity against all cell stages ${ }^{47}$ For traditional/herbal medicines, the anti-annexin A2 antibody-conjugated PLGA nanoparticle delivering 
curcumin was shown to significantly increase compound cellular uptake by human breast adenocarcinoma cells (MDA-MB-231). ${ }^{64}$

\section{Aptamers}

Aptamers are short single-stranded DNA or RNA sequences that can fold to three-dimensional structure and bind to specific targets on the cancer cell surfaces that express specific targets for different aptamers. For instance, nucleolin receptor is specific for AS14111 aptamer and EpCAM protein is specific for EpCAM aptamer. The aptamer AS14111-conjugated PEG-PLGA nanoparticle delivering gemcitabine to target nucleolin receptor was shown to increase drug cellular uptake (36\%) by nucleolin-overexpressing human lung cancer cells (A549) compared with non-targeted nanoparticles, with enhanced cytotoxic activity $\left(\mathrm{IC}_{50}: 4.9 \mu \mathrm{g} / \mathrm{mL}\right.$ and $28.9 \mu \mathrm{g} / \mathrm{mL}$, respectively). ${ }^{49}$ The AS14111-conjugated undecylenic acid modified, thermally hydrocarbonized porous silicon (UnTHCPSi) nanoparticle delivering methotrexate was shown to increase drug cellular uptake by nucleolin-overexpressing human breast adenocarcinoma cells (MDA-MB-231) with enhanced cytotoxic activity compared with non-targeted nanoparticles and nucleolin-negative fibroblasts cells (NIH 3T3). ${ }^{50}$ The aptamer AS14111-conjugated polymersome delivering doxorubicin was shown to increase drug cellular uptake by nucleolin-overexpressing human breast adenocarcinoma cells (MCF-7) with enhanced cytotoxic activity compared with mutated aptamer-conjugated nanoparticles (mean $\mathrm{IC}_{50}: 210.9 \mathrm{ng} / \mathrm{mL}$ and $369.4 \mathrm{ng} / \mathrm{mL}$, respectively). In addition, it also produced significant tumor growth inhibition in MCF-7 tumor-bearing mice compared with mutated aptamer-conjugated nanoparticles. ${ }^{52}$ For traditional/herbal medicines, the EpCAM aptamer-conjugated lipid-polymerlecithin hybrid delivering curcumin was shown to increase compound cellular uptake by EpCAM-overexpressing human colon adenocarcinoma cells (HT29) compared with EpCAMnegative human embryonic kidney cells (HEK293T), $\left(58.9 \% \pm 2.6 \%\right.$ and $72.4 \% \pm 1.3 \%$, respectively. ${ }^{65}$

\section{Carbohydrates or polysaccharides}

Galactose is one of targeting ligands in group of carbohydrates that is widely used as a targeting agent for nanoparticles. It is specifically recognized by the asialoglycoprotein receptor (ASGPR), which is overexpressed on liver cancer cell surface. ${ }^{54}$ The galactose-conjugated lithocholic acidPEG-lactobionic acid nanoparticles delivering doxorubicin was shown to increase drug cellular uptake by human liver cancer cells (SK-HEP-1) leading to massive cell death and tumor growth inhibition compared with non-targeted nanoparticles. ${ }^{54}$ The galactose-conjugated pectin nanoparticle delivering 5-fluorouracil was shown to increase drug cellular uptake by human hepatocellular liver carcinoma cells (HepG2) with enhanced cytotoxic activity compared with free drug (mean $\mathrm{IC}_{50}: 0.17 \times 10^{-4} \mathrm{~mol} / \mathrm{L}$ and $0.45 \times 10^{-4} \mathrm{~mol} / \mathrm{L}$, respectively). Moreover, the targeted nanoparticle also improved pharmacokinetic profile of the drugs. ${ }^{55}$ On the other hand, the lactose-conjugated nanoparticle delivering doxorubicin was shown to improve drug efficacy, but not as good as galactose. ${ }^{53}$ The galactose conjugates not only specifically bind to ASGPR but also to lectin receptor, which is overexpressed on the alveolar macrophages, liver endothelial Kupffer cells, splenic macrophages, peritoneal macrophages, and macrophages of brain. The galactose-conjugated solid lipid nanoparticles delivering doxorubicin specifically targeted human lung cancer cells (A549) resulting in higher cellular uptake, enhanced cytotoxic activity, and improved pharmacokinetic profiles compared with non-targeted nanoparticles and free drug. ${ }^{57}$

\section{Controlled drug release of active targeting nanoparticles}

Controlled drug release is a property of drug delivery systems in cancer therapy. Drugs are delivered and released at specific location to avoid side effects to normal cells. ${ }^{72}$ Most studies included in this review showed biphasic characteristics of drugs released from both targeted and non-targeted nanoparticles, ie, initial burst release, followed by sustained release. For instance, about $48 \%$ and $46 \%$ of gemcitabine were released from folic acid-conjugated bovine serum albumin nanoparticles and non-targeted nanoparticles during the first 2 hours, respectively. Sustained release of up to $99 \%$ and $94 \%$ was observed at 36 hours and $\mathrm{pH} 7.4$ after burst release of targeted and non-targeted nanoparticles, respectively. ${ }^{38}$ About $22 \%$ and $29 \%$ of doxorubicin were shown to release from galactose-conjugated solid lipid nanoparticles and nontargeted nanoparticles during the first 8 hours, respectively. After burst release, sustained released was observed up to $76 \%$ and $93 \%$ at $144 \mathrm{~h}$ and $\mathrm{pH} 7.4$ for targeted and nontargeted nanoparticles, respectively. ${ }^{57}$ Moreover, in some cases, the amount of drug released from nanoparticles at endolysosomal environment ( $\mathrm{pH}$ 5.5) or cancer cell environment ( $\mathrm{pH}$ 6.8) was shown to be higher than that from physiological environment ( $\mathrm{pH} 7.4$ ). Up to $60 \%$ of doxorubicin was released from anti-CD20-conjugated active carbon nanoparticles and non-targeted nanoparticles at 12 hours and at $\mathrm{pH}$ 5.5. At $\mathrm{pH} \mathrm{7.4,} \mathrm{on} \mathrm{the} \mathrm{other} \mathrm{hand,} \mathrm{the} \mathrm{release}$ 
from nanoparticles was only $20 \%{ }^{45}$ Similarly, about $28 \%$ and $24 \%$ of gemcitabine burst were released during the first 24 hours from AS1411 aptamer-conjugated PEG-PLGA nanoparticles and non-targeted nanoparticles, respectively. After burst release, up to $44 \%$ and $41 \%$ sustained release were observed in both formulations at 120 hours and $\mathrm{pH} 5.5$ for targeted and non-targeted nanoparticles, respectively. Only $30 \%$ release was observed at $\mathrm{pH} 7.4 .{ }^{49}$ In another study, doxorubicin released from chondroitin sulfate A-deoxycholic acid at day 6 was $92 \%, 53 \%$, and $34 \%$ for $\mathrm{pH} 5.5,6.8$, and 7.4 , respectively. ${ }^{60}$ These results suggested that conjugation of targeting ligands on the surface of nanoparticles did not affect drug release from nanoparticles. Furthermore, higher amount of drug released at acidic $\mathrm{pH}$ would benefit the delivery of cancer chemotherapeutic agents to cancer cells with lower side effects to normal cells.

\section{Conclusion}

Active targeting nanoparticles of chemotherapeutic drugs or traditional/herbal medicines have been demonstrated in various studies both in vitro and in vivo to improve selectivity of cellular uptake of drugs to cancer cells through receptormediated endocytosis and/or cytotoxicity. They provide several advantages over the conventional chemotherapeutic drugs and non-targeted nanoparticle platform, particularly in regard to enhancement of drug efficacy and safety. Active targeting nanoparticles possess several advantages in cancer therapy including enhancement of selectivity of drugs to cancer cells to avoid side effects to normal cells, enhancement of drug accumulation and anticancer activity in cancer cells, and efficiency in control of drug release. Nevertheless, some disadvantages of active targeting nanoparticles include their limitation of clinical uses in only certain types of cancer that express specific receptors on the cell surfaces. Moreover, manufacturing of nanoparticle platforms is costly and requires sophisticated technology. Selection of the types of targeting nanoparticles is determined by the types of target proteins or receptors expressed on cancer cell surfaces. Clinical studies are required to confirm their application in cancer patients.

\section{Acknowledgments}

The authors would like to thank the Center of Excellence in Pharmacology and Molecular Biology of Malaria and Cholangiocarcinoma, Chulabhorn International College of Medicine at Thammasat University, Rangsit Center, for providing all the necessary support in conducting this systematic review.

\section{Disclosure}

The authors report no conflicts of interest in this work.

\section{References}

1. Siegel RL, Miller KD, Jemal A. Cancer statistics, 2017. CA Cancer J Clin. 2017;67(1):7-30.

2. Kuppens IE, Bosch TM, van Maanen MJ, et al. Oral bioavailability of docetaxel in combination with OC144-093 (ONT-093). Cancer Chemother Pharmacol. 2005;55(1):72-78.

3. Vandana M, Sahoo SK. Long circulation and cytotoxicity of PEGylated gemcitabine and its potential for the treatment of pancreatic cancer. Biomaterials. 2010;31(35):9340-9356.

4. Yu H, Tang Z, Zhang D, et al. Pharmacokinetics, biodistribution and in vivo efficacy of cisplatin loaded poly(L-glutamic acid)-g-methoxy poly(ethylene glycol) complex nanoparticles for tumor therapy. J Control Release. 2015;205:89-97.

5. Cho K, Wang X, Nie S, Chen ZG, Shin DM. Therapeutic nanoparticles for drug delivery in cancer. Clin Cancer Res. 2008;14(5):1310-1316.

6. Zhuang Y, Deng H, Su Y, et al. Aptamer-functionalized and backbone redox-responsive hyperbranched polymer for targeted drug delivery in cancer therapy. Biomacromolecules. 2016;17(6):2050-2062.

7. Wu G, Wang Z, Bian X, Du X, Wei C. Folate-modified doxorubicinloaded nanoparticles for tumor-targeted therapy. Pharm Biol. 2014; 52(8):978-982.

8. Qi X, Fan Y, He H, Wu Z. Hyaluronic acid-grafted polyamidoamine dendrimers enable long circulation and active tumor targeting simultaneously. Carbohydr Polym. 2015;126:231-239.

9. Wang Y, Song D, Costanza F, et al. Targeted delivery of tanshinone IIA-conjugated mPEG-PLGA-PLL-cRGD nanoparticles to hepatocellular carcinoma. J Biomed Nanotechnol. 2014;10(11):3244-3252.

10. Zhao Y, Lin D, Wu F, et al. Discovery and in vivo evaluation of novel RGD-modified lipid-polymer hybrid nanoparticles for targeted drug delivery. Int J Mol Sci. 2014;15(10):17565-17576.

11. Yang B, Ni X, Chen L, et al. Honokiol-loaded polymeric nanoparticles: an active targeting drug delivery system for the treatment of nasopharyngeal carcinoma. Drug Deliv. 2017;24(1):660-669.

12. Gray BP, McGuire MJ, Brown KC. A liposomal drug platform overrides peptide ligand targeting to a cancer biomarker, irrespective of ligand affinity or density. PLoS One. 2013;8(8):e72938.

13. Gao H, Zhang S, Yang Z, Cao S, Jiang X, Pang Z. In vitro and in vivo intracellular distribution and anti-glioblastoma effects of docetaxelloaded nanoparticles functioned with IL-13 peptide. Int J Pharm. 2014;466(1-2):8-17.

14. Namgung R, Mi Lee Y, Kim J, et al. Poly-cyclodextrin and polypaclitaxel nano-assembly for anticancer therapy. Nat Commun. 2014; 5:3702.

15. Yang N, Jiang Y, Zhang H, et al. Active targeting docetaxel-PLA nanoparticles eradicate circulating lung cancer stem-like cells and inhibit liver metastasis. Mol Pharm. 2015;12(1):232-239.

16. Hong M, Zhu S, Jiang Y, et al. Novel anti-tumor strategy: PEGhydroxycamptothecin conjugate loaded transferrin-PEG-nanoparticles. J Control Release. 2010;141(1):22-29.

17. Nag M, Gajbhiye V, Kesharwani P, Jain NK. Transferrin functionalized chitosan-PEG nanoparticles for targeted delivery of paclitaxel to cancer cells. Colloids Surf B Biointerfaces. 2016;148:363-370.

18. Jiang X, Sha X, Xin H, et al. Self-aggregated pegylated poly (trimethylene carbonate) nanoparticles decorated with c(RGDyK) peptide for targeted paclitaxel delivery to integrin-rich tumors. Biomaterials. 2011;32(35):9457-9469.

19. Nazli C, Demirer GS, Yar Y, Acar HY, Kizilel S. Targeted delivery of doxorubicin into tumor cells via MMP-sensitive PEG hydrogelcoated magnetic iron oxide nanoparticles (MIONPs). Colloids Surf B Biointerfaces. 2014;122:674-683.

20. Gao Y, Zhou Y, Zhao L, et al. Enhanced antitumor efficacy by cyclic RGDyK-conjugated and paclitaxel-loaded $\mathrm{pH}$-responsive polymeric micelles. Acta Biomater. 2015;23:127-135. 
21. Hu G, Zhang H, Zhang L, Ruan S, He Q, Gao H. Integrin-mediated active tumor targeting and tumor microenvironment response dendrimergelatin nanoparticles for drug delivery and tumor treatment. Int J Pharm. 2015;496(2):1057-1068.

22. Kulhari H, Pooja D, Shrivastava S, Naidu VGM, Sistla R. Peptide conjugated polymeric nanoparticles as a carrier for targeted delivery of docetaxel. Colloids Surf B Biointerfaces. 2014;117:166-173.

23. Liu CW, Lin WJ. Polymeric nanoparticles conjugate a novel heptapeptide as an epidermal growth factor receptor-active targeting ligand for doxorubicin. Int J Nanomedicine. 2012;7:4749-4767.

24. Taheri A, Dinarvand R, Atyabi F, et al. Enhanced anti-tumoral activity of methotrexate-human serum albumin conjugated nanoparticles by targeting with Luteinizing Hormone-Releasing Hormone (LHRH) peptide. Int J Mol Sci. 2011;12(7):4591-4608.

25. Hu G, Chun X, Wang Y, He Q, Gao H. Peptide mediated active targeting and intelligent particle size reduction-mediated enhanced penetrating of fabricated nanoparticles for triple-negative breast cancer treatment. Oncotarget. 2015;6(38):41258-41274.

26. Cai L, Qiu N, Li X, et al. A novel truncated basic fibroblast growth factor fragment-conjugated poly (ethylene glycol)-cholesterol amphiphilic polymeric drug delivery system for targeting to the FGFR-overexpressing tumor cells. Int J Pharm. 2011;408(1-2):173-182.

27. Yin S, Huai J, Chen X, et al. Intracellular delivery and antitumor effects of a redox-responsive polymeric paclitaxel conjugate based on hyaluronic acid. Acta Biomater. 2015;26:274-285.

28. Suh MS, Shen J, Kuhn LT, Burgess DJ. Layer-by-layer nanoparticle platform for cancer active targeting. Int J Pharm. 2017;517(1-2): 58-66.

29. Freag MS, Elnaggar YS, AbdelmonsifDA, Abdallah OY. Layer-by-layercoated lyotropic liquid crystalline nanoparticles for active tumor targeting of rapamycin. Nanomedicine (Lond). 2016;11(22):2975-2996.

30. Upadhyay KK, Mishra AK, Chuttani K, et al. The in vivo behavior and antitumor activity of doxorubicin-loaded poly ( $\gamma$-benzyl l-glutamate)block-hyaluronan polymersomes in Ehrlich ascites tumor-bearing BalB/c mice. Nanomedicine. 2012;8(1):71-80.

31. Mizrahy S, Goldsmith M, Leviatan-Ben-Arye S, et al. Tumor targeting profiling of hyaluronan-coated lipid based-nanoparticles. Nanoscale. 2014;6(7):3742-3752.

32. Xiong H, Du S, Ni J, Zhou J, Yao J. Mitochondria and nuclei dualtargeted heterogeneous hydroxyapatite nanoparticles for enhancing therapeutic efficacy of doxorubicin. Biomaterials. 2016;94:70-83.

33. Cho HJ, Yoon IS, Yoon HY, et al. Polyethylene glycol-conjugated hyaluronic acid-ceramide self-assembled nanoparticles for targeted delivery of doxorubicin. Biomaterials. 2012;33(4):1190-1200.

34. Zhong Y, Zhang J, Cheng R, et al. Reversibly crosslinked hyaluronic acid nanoparticles for active targeting and intelligent delivery of doxorubicin to drug resistant CD44+ human breast tumor xenografts. J Control Release. 2015;205:144-154.

35. Sun B, Deng C, Meng F, Zhang J, Zhong Z. Robust, active tumortargeting and fast bioresponsive anticancer nanotherapeutics based on natural endogenous materials. Acta Biomater. 2016;45:223-233.

36. Tao W, Zhang J, Zeng X, et al. Blended nanoparticle system based on miscible structurally similar polymers: a safe, simple, targeted, and surprisingly high efficiency vehicle for cancer therapy. Adv Healthc Mater. 2015;4(8):1203-1214.

37. Cheng L, Hu Q, Cheng L, et al. Construction and evaluation of PAMAM-DOX conjugates with superior tumor recognition and intracellular acid-triggered drug release properties. Colloids Surf B Biointerfaces. 2015;136:37-45.

38. Dubey RD, Alam N, Saneja A, et al. Development and evaluation of folate functionalized albumin nanoparticles for targeted delivery of gemcitabine. Int J Pharm. 2015;492(1-2):80-91.

39. Ji J, Zuo P, Wang YL. Enhanced antiproliferative effect of carboplatin in cervical cancer cells utilizing folate-grafted polymeric nanoparticles. Nanoscale Res Lett. 2015;10(1):453.

40. Ye WL, Du JB, Zhang BL, et al. Cellular uptake and antitumor activity of DOX-hyd-PEG-FA nanoparticles. PLoS One. 2014;9(5):e97358.
41. Morelli C, Maris P, Sisci D, et al. PEG-templated mesoporous silica nanoparticles exclusively target cancer cells. Nanoscale. 2011;3(8):3198-3207.

42. Yin JJ, Sharma S, Shumyak SP, et al. Synthesis and biological evaluation of novel folic acid receptor-targeted, $\beta$-cyclodextrin-based drug complexes for cancer treatment. PLoS One. 2013;8(5):e62289.

43. Liang C, Yang Y, Ling Y, Huang Y, Li T, Li X. Improved therapeutic effect of folate-decorated PLGA-PEG nanoparticles for endometrial carcinoma. Bioorg Med Chem. 2011;19(13):4057-4066.

44. McCarron PA, Marouf WM, Quinn DJ, et al. Antibody targeting of camptothecin-loaded PLGA nanoparticles to tumor cells. Bioconjug Chem. 2008;19(8):1561-1569.

45. Jiang S, Wang X, Zhang Z, et al. CD20 monoclonal antibody targeted nanoscale drug delivery system for doxorubicin chemotherapy: an in vitro study of cell lysis of CD20-positive Raji cells. Int $J$ Nanomedicine. 2016;11:5505-5518. eCollection 2016.

46. Trabulo S, Aires A, Aicher A, Heeschen C, Cortajarena AL. Multifunctionalized iron oxide nanoparticles for selective targeting of pancreatic cancer cells. Biochim Biophys Acta. 2017;1861(6):1597-1605.

47. Acharya S, Dilnawaz F, Sahoo SK. Targeted epidermal growth factor receptor nanoparticle bioconjugates for breast cancer therapy. Biomaterials. 2009;30(29):5737-5750.

48. Kouchakzadeh H, Shojaosadati SA, Mohammadnejad J, Paknejad M, Rasaee MJ. Attachment of an anti-MUC1 monoclonal antibody to 5-FU loaded BSA nanoparticles for active targeting of breast cancer cells. Hum Antibodies. 2012;21(3-4):49-56.

49. Alibolandi M, Ramezani M, Abnous K, Hadizadeh F. AS1411 Aptamerdecorated biodegradable polyethylene glycol-poly(lactic-co-glycolic acid) nanopolymersomes for the targeted delivery of gemcitabine to nonsmall cell lung cancer in vitro. J Pharm Sci. 2016;105(5):1741-1750.

50. Zhang F, Correia A, Mäkilä E, et al. Receptor-mediated surface charge inversion platform based on porous silicon nanoparticles for efficient cancer cell recognition and combination therapy. ACS Appl Mater Interfaces. 2017;9(11):10034-10046.

51. Xu G, Yu X, Zhang J, et al. Robust aptamer-polydopamine-functionalized M-PLGA-TPGS nanoparticles for targeted delivery of docetaxel and enhanced cervical cancer therapy. Int J Nanomedicine. 2016;11: 2953-2965.

52. Li X, Zhu X, Qiu L. Constructing aptamer anchored nanovesicles for enhanced tumor penetration and cellular uptake of water soluble chemotherapeutics. Acta Biomater. 2016;35:269-279.

53. Mou Q, Ma Y, Zhu X, Yan D. A small molecule nanodrug consisting of amphiphilic targeting ligand-chemotherapy drug conjugate for targeted cancer therapy. J Control Release. 2016;230:34-44.

54. Singh B, Jang Y, Maharjan S, et al. Combination therapy with doxorubicin-loaded galactosylated poly(ethyleneglycol)-lithocholic acid to suppress the tumor growth in an orthotopic mouse model of liver cancer. Biomaterials. 2017;116:130-144.

55. Yu CY, Wang YM, Li NM, et al. In vitro and in vivo evaluation of pectin-based nanoparticles for hepatocellular carcinoma drug chemotherapy. Mol Pharm. 2014;11(2):638-644.

56. Liang HF, Chen SC, Chen MC, Lee PW, Chen CT, Sung HW. Paclitaxelloaded poly(gamma-glutamic acid)-poly(lactide) nanoparticles as a targeted drug delivery system against cultured HepG2 cells. Bioconjug Chem. 2006;17(2):291-299.

57. Jain A, Kesharwani P, Garg NK, et al. Galactose engineered solid lipid nanoparticles for targeted delivery of doxorubicin. Colloids Surf B Biointerfaces. 2015;134:47-58.

58. Sandoval MA, Sloat BR, Lansakara-P DS, et al. EGFR-targeted stearoyl gemcitabine nanoparticles show enhanced anti-tumor activity. J Control Release. 2012;157(2):287-296.

59. Talelli M, Oliveira S, Rijcken CJ, et al. Intrinsically active nanobodymodified polymeric micelles for tumor-targeted combination therapy. Biomaterials. 2013;34(4):1255-1260

60. Lee JY, Chung SJ, Cho HJ, Kim DD. Bile acid-conjugated chondroitin sulfate A-based nanoparticles for tumor-targeted anticancer drug delivery. Eur J Pharm Biopharm. 2015;94:532-541. 
61. Chen C, Hu H, Qiao M, et al. Anti-tumor activity of paclitaxel through dual-targeting lipoprotein-mimicking nanocarrier. J Drug Target. 2015;23(4):311-322.

62. Zhang L, Yao J, Zhou J, Wang T, Zhang Q. Glycyrrhetinic acid-grafthyaluronic acid conjugate as a carrier for synergistic targeted delivery of antitumor drugs. Int J Pharm. 2013;441(1-2):654-664.

63. Kesharwani P, Banerjee S, Padhye S, Sarkar FH, Iyer AK. Hyaluronic acid engineered nanomicelles loaded with 3,4-difluorobenzylidene curcumin for targeted killing of CD44+ stem-like pancreatic cancer cells. Biomacromolecules. 2015;16(9):3042-3053.

64. Thamake SI, Raut SL, Ranjan AP, Gryczynski Z, Vishwanatha JK. Surface functionalization of PLGA nanoparticles by non-covalent insertion of a homo-bifunctional spacer for active targeting in cancer therapy. Nanotechnology. 2011;22(3):035101.

65. Li L, Xiang D, Shigdar S, et al. Epithelial cell adhesion molecule aptamer functionalized PLGA-lecithin-curcumin-PEG nanoparticles for targeted drug delivery to human colorectal adenocarcinoma cells. Int J Nanomedicine. 2014;9:1083-1096.

66. Jeong JY, Hong EH, Lee SY, et al. Boronic acid-tethered amphiphilic hyaluronic acid derivative-based nanoassemblies for tumor targeting and penetration. Acta Biomater. 2017;53:414-426.
67. Bazak R, Houri M, El Achy S, Kamel S, Refaat T. Cancer active targeting by nanoparticles: a comprehensive review of literature. J Cancer Res Clin Oncol. 2015;141(5):769-784.

68. Cornelio DB, Roesler R, Schwartsmann G. Gastrin-releasing peptide receptor as a molecular target in experimental anticancer therapy. Ann Oncol. 2007;18(9):1457-1466.

69. Master AM, Sen Gupta A. EGF receptor-targeted nanocarriers for enhanced cancer treatment. Nanomedicine (Lond). 2012;7(12):1895-1906.

70. Kapoor A, Kumar S. Cancer stem cell: a rogue responsible for tumor development and metastasis. Indian J Cancer. 2014;51(3): 282-289.

71. Zwicke GL, Mansoori GA, Jeffery CJ. Utilizing the folate receptor for active targeting of cancer nanotherapeutics. Nano Reviews. 2012;3:10.3402/nano.v3403i3400.18496.

72. Rodzinski A, Guduru R, Liang P, et al. Targeted and controlled anticancer drug delivery and release with magnetoelectric nanoparticles. Sci Rep. 2016;6:20867.
International Journal of Nanomedicine

\section{Publish your work in this journal}

The International Journal of Nanomedicine is an international, peerreviewed journal focusing on the application of nanotechnology in diagnostics, therapeutics, and drug delivery systems throughout the biomedical field. This journal is indexed on PubMed Central, MedLine, CAS, SciSearch $®$, Current Contents $\AA /$ Clinical Medicine,

\section{Dovepress}

Journal Citation Reports/Science Edition, EMBase, Scopus and the Elsevier Bibliographic databases. The manuscript management system is completely online and includes a very quick and fair peer-review system, which is all easy to use. Visit http://www.dovepress.com/ testimonials.php to read real quotes from published authors. 